\title{
Direct URCA Processes in Supernovae and Accretion Disks with Arbitrary Magnetic Field
}

\author{
Igor Ognev ${ }^{1, \star}$ \\ ${ }^{1}$ P. G. Demidov Yaroslavl State University, Sovietskaya 14, 150003 Yaroslavl, Russia
}

\begin{abstract}
An effect of a magnetic field of an arbitrary strength on the beta-decay and reactions related with it by the crossing symmetry (the beta-processes) in supernovae and accretion disks around black holes is analyzed. Rates of the beta-processes and the energy and momentum transfered through them to an optically transparent matter are calculated. It is shown that the macroscopic momentum transferred to the medium increases linearly with the magnetic field strength and can substantially affect the dynamics of supernovae and accretion disks especially when a matter inside is degenerate. It is also demonstrated that the rates of the beta-processes and the energy deposition in these reactions for the magnetic field strength $B \lesssim 10^{15} \mathrm{G}$, which is assumed to be typical in supernovae and accretion disks, are lower than in the absence of the field. This suppression is more pronounced for reactions with neutrinos.
\end{abstract}

\section{Introduction}

Neutrinos can strongly affect the processes occurring in many astrophysical objects, especially explosive processes accompanied by the release of a large amount of energy and heating of the surrounding medium. Such processes include the core collapse supernova which is the final stage in an evolution of massive stars as well as a merger of close binary systems. Despite the fact that both these phenomena are fundamentally different, their common features are the powerful neutrino radiation and possible connection with cosmological gamma-ray bursts [1] which remain an unsolved problem in theoretical astrophysics for many years. In a supernova explosion, a source of the neutrino emission is the proto-neutron star that forms as the result of the core collapse of a massive star at the final stage of its evolution. It should be noted that neutrinos are the main engine leading to a successive explosion of supernovae. A merger of stars in a close binary system leads to the formation of a central object (as a rule, it is a black hole or, very rarely, a massive neutron star) and a hot accretion disk around it. In this case, the disk is a source of powerful fluxes of neutrinos. Note that an analogous system consisting of a central black hole and accretion disk can be formed during a failed supernova explosion called the collapsar model [2]. In this model, a black hole is appeared as the result of the core collapse of a massive star, rather than a proto-neutron star. The absence of a powerful neutrino radiation from the central part leads to the accretion of the remaining matter to the black hole and formation of a hot disk around the hole. General interest in such systems is connected with the fact that a large amount of energetic neutrino is emitted from the hot disk and produces a relativistic electron-positron plasma

^e-mail: ognev@uniyar.ac.ru 
which could be a source of gamma-ray bursts [1]. However, the merger of compact objects (neutron stars or black holes) apparently leads to the formation of short-duration gamma-ray bursts [3]. Longduration gamma-ray bursts are usually associated with the supernova explosions with a large energy release and probably with collapsars [1]. It should be noted that the merger of a compact object with the massive Wolf-Rayet star can also be the source of the long-duration gamma-ray bursts [4].

The dense and hot medium that forms after a supernova explosion, as well as the merger of stars in a close binary system, is not transparent to photons, and emission of neutrinos is the main channel for its cooling. Due to high temperatures, the medium predominantly consists of free nucleons, photons, and relativistic electrons and positrons being in the local thermodynamic equilibrium. Depending on the specific conditions, neutrinos in such a medium can either be in equilibrium, like in supernova cores, or be freely emitted, like in accretion disks or envelopes of supernovae. The main neutrino reactions in such media are the beta-processes (called often the direct URCA-processes) [5]:

$$
\begin{aligned}
& p+e^{-} \rightleftarrows n+v_{e}, \\
& n+e^{+} \rightleftarrows p+\bar{v}_{e}, \\
& n \rightleftarrows p+e^{-}+\bar{v}_{e},
\end{aligned}
$$

which are important primarily as the main channel of the energy exchange between the medium and neutrinos in the case of supernovae and the energy source of gamma-ray bursts in the merger of stars in the close binary system. An important feature in such astrophysical cataclysms is that a strong magnetic field can be generated. Simulations show that the magnetic field strength in supernovae [6, 7] and in accretion disks $[4,8]$ can reach the values $B \sim 10^{15} \mathrm{G}$. Such a strong magnetic field not only significantly affects the dynamics of supernovae and accretion disks but also modifies the interaction of neutrinos with their matter (see, for example, [9-11]).

In this paper, the modifications of the beta-processes taking place in supernovae and accretion disks, by the magnetic field of an arbitrary strength are discussed. The results of calculations of the neutrino- and antineutrino-production rates and the energy-momentum deposition to optically transparent medium are presented. A dependence of these quantities on the magnetic field strength and parameters of the medium is demonstrated. Other details of the processes presented here can be found in the recent paper [12].

In this paper we use the system of units in which $c=\hbar=k=1$, where $c$ is the velocity of light, $\hbar$ is the Planck constant, and $k$ is the Boltzmann constant.

\section{General formalism}

The macroscopic quantities, being the most interesting for astrophysical applications, are the reaction rate (the number of processes occurring in a unit volume per unit time):

$$
\Gamma=\frac{1}{V} \int \sum_{i, f} \frac{\left|S_{i f}\right|^{2}}{\mathcal{T}} \prod_{i, f} f_{i}\left(1-f_{f}\right) d n_{i} d n_{f},
$$

and the energy and momentum transferred from neutrinos to a unit volume of the medium per unit time:

$$
Q^{\mu}=(Q, \overrightarrow{\mathcal{F}})= \pm \frac{1}{V} \int \sum_{i, f} q^{\mu} \frac{\left|S_{i f}\right|^{2}}{\mathcal{T}} \prod_{i, f} f_{i}\left(1-f_{f}\right) d n_{i} d n_{f}
$$

where $V$ is the normalization volume, $\left|S_{i f}\right|^{2} / \mathcal{T}$ is the transition probability from the initial (i) state to final $(f)$ one per unit time, $q^{\mu}=(\omega, \vec{q})$ is the neutrino four-momentum, and the sign " \pm " in (5) specifies 
either absorption or emission neutrino process is considered. The summation and integration is carried out over the phase space $d n_{i, f}$ of all particles taking into account their distribution functions $f_{i, f}$ (it is implicitly assumed that all final particles are fermions). It is convenient to separate the integration over the neutrino momentum from all other. One can define the quantity called the emissivity [12]:

$$
\mathcal{K}(q)=\sum_{i, f \neq \nu} \int \frac{\left|S_{i f}\right|^{2}}{\mathcal{T}} \prod_{i, f \neq v} f_{i}\left(1-f_{f}\right) d n_{i} d n_{f},
$$

where the reaction with neutrino in the final state is assumed. It completely determines the property of the matter to emit or absorb the neutrino with the four-momentum $q$ in the transition $i \leftrightarrow f$.

Under conditions of supernovae and accretion disks, the total emissivity for neutrino and antineutrino in beta-processes can be presented in the simple analytical form [12]:

$$
\begin{gathered}
\mathcal{K}_{v}(q)=K_{0} \Phi\left(b, x_{v}, \beta_{v}\right) \frac{e^{(\delta \chi+\Delta) / t-a}}{e^{x_{v} / t-a}+1}, \\
\mathcal{K}_{\bar{v}}(q)=K_{0} \Phi\left(b, x_{\bar{v}}, \beta_{\bar{v}}\right) \frac{1}{e^{x_{\bar{v}} / t+a}+1}, \\
K_{0}=\frac{\left(g_{v}^{2}+3 g_{a}^{2}\right) \cos ^{2} \theta_{c}}{2 \pi} G_{F}^{2} N_{n} m_{e}^{2}, \\
\Phi(x, b, \beta)=2 x \sqrt{x^{2}-1} \theta(x-\sqrt{1+2 b})+ \\
+\frac{b x}{\sqrt{x^{2}-1}}[\theta(x-1)-\theta(x-\sqrt{1+2 b})]-g_{v a} \frac{b x \cos \beta}{\sqrt{x^{2}-1}} \theta(x-1),
\end{gathered}
$$

where $m_{n}, m_{p}, m_{e}$ and $\mu_{n}, \mu_{p}, \mu_{e}$ are the masses and local chemical potentials of neutrons, protons and electrons, $\Delta=\left(m_{n}-m_{p}\right) / m_{e}, \delta \chi=\left(\mu_{e}+\mu_{p}-\mu_{n}\right) / m_{e}, T$ is the local temperature of the medium, $t=T / m_{e}, a=\mu_{e} / T, N_{n}$ is the neutrons number density, $x_{v, \bar{v}}=\omega_{v, \bar{v}} / m_{e} \pm \Delta$ are the scaled neutrino and antineutrino energy, $b=e B / m_{e}^{2}=B / B_{0}$ is the magnetic field strength measured in units of the Schwinger value $B_{0} \approx 4.41 \times 10^{13} \mathrm{G}, e>0$ is the elementary charge, $\beta_{v, \bar{v}}$ is the angle between the (anti)neutrino momentum and magnetic field direction. Here, the following constants are used [13]: $g_{v} \approx 1, g_{a} \approx-1.27$ are the vector and axial constants of the nucleon charged current, $g_{v a}=\left(g_{a}^{2}-\right.$ $\left.g_{v}^{2}\right) /\left(3 g_{a}^{2}+g_{v}^{2}\right) \approx 0.11, \theta_{c}$ is the Cabibbo angle, $\cos \theta_{c} \approx 0.97, G_{F} \approx 1.17 \times 10^{-11} \mathrm{MeV}^{-2}$ is the Fermi constant. The dependence on the magnetic-field strength is entering in $\mathcal{K}_{v}(q)$ and $\mathcal{K}_{\bar{v}}(q)$ through the function $\Phi(x, b, \beta)$ only. The first term in this function coincides with the one appeared in the absence of the field and describes the magnetized matter ability to emit and absorb neutrinos with high energies $(x \geqslant \sqrt{1+2 b})$. The second term in $\Phi(x, b, \beta)$ dominates in the limit of the strong magnetic field and responsible for the low-energy neutrino spectrum $(x \leqslant \sqrt{1+2 b})$. Note that this term is the result of exact calculations [12] of neutrino and antineutrino emissivities for the beta-processes under the supernova and accretion disk conductions. The last term in $\Phi(x, b, \beta)$ is linear in $\cos \beta$ and determines the asymmetric part of the emissivity. It existence leads to an uncompensated momentum transfered to the medium along the magnetic field direction.

\section{Medium transparent for neutrino}

The case of a medium transparent for neutrinos is the most simple for calculations. However, it is quite applicable in astrophysics. In particular, the matter of accretion disks and an external part of supernova envelopes is transparent for neutrinos and antineutrinos and their distribution functions can 
be well approached by unit, $f_{v, \bar{v}} \ll 1$, in (4) and (5). In this case, the neutrino- and antineutrinoproduction number and the energy emitted from the unit volume of the medium per unit time in the beta-processes can be written as follows:

$$
\begin{aligned}
\Gamma_{\nu, \bar{v}} & =\frac{1}{(2 \pi)^{3}} \int \mathcal{K}_{v, \bar{\nu}}(q) d^{3} q, \\
Q_{v, \bar{v}} & =\frac{1}{(2 \pi)^{3}} \int \omega \mathcal{K}_{v, \bar{v}}(q) d^{3} q .
\end{aligned}
$$

As noted above, the existence of the asymmetric part in the emissivity (the third term in $\Phi(x, b, \beta)$ in Eq. (7)) leads to a macroscopic momentum transferred to the medium. In the case of the transparent for (anti)neutrino matter only the component along the magnetic field direction is non zero:

$$
\mathcal{F}_{v, \bar{v} \|}=-\frac{1}{(2 \pi)^{3}} \int \omega \cos \beta \mathcal{K}_{v, \bar{v}}(q) d^{3} q .
$$

This quantity becomes quite simple in the case of the ultrarelativistic electron-positron plasma which exists in supernovae and accretion disks. In this limit, the force density along the magnetic field arising from neutrino and antineutrino emission has the form [12]:

$$
\begin{gathered}
\mathcal{F}_{v \|}=\mathcal{F}_{0} I_{3}(a) Y /(1+Y), \quad \mathcal{F}_{\bar{v} \|}=\mathcal{F}_{0} I_{3}(-a) /(1+Y), \\
\mathcal{F}_{0}=\frac{\left(g_{a}^{2}-g_{v}^{2}\right) \cos ^{2} \theta_{c}}{12 \pi^{3}} G_{F}^{2} e B T^{4} \frac{\rho}{m_{N}}, \quad I_{3}(a)=\int_{0}^{\infty} \frac{y^{3} d y}{e^{y-a}+1},
\end{gathered}
$$

where $Y=N_{p} / N_{n}$ is the proton-to-neutron number density ratio, $\rho$ is the density of the matter, $m_{N} \approx$ $940 \mathrm{MeV}$ is the nucleon mass [13]. Here, $\mathcal{F}_{0} \approx 1.85 \times 10^{14} b t^{4} \rho_{12} \mathrm{dyn} / \mathrm{cm}^{3}$ gives the typical value for the force density in the nondegenerate electron-positron plasma with $\rho_{12}=\rho / 10^{12} \mathrm{~g} / \mathrm{cm}^{3}$. In the limit of large values of $a$, the integral $I_{3}(a)$ in (10) can be approximated by the following expressions [12]:

$$
I_{3}(a) \approx \frac{a^{4}}{4}+\frac{\pi^{2}}{2} a^{2}+3.2 a+\frac{7 \pi^{4}}{120}, \quad I_{3}(-a) \approx 6 e^{-a} .
$$

So, for large values of the electron degeneracy parameter $a=\mu_{e} / T$ the momentum transferred to the medium increases by the factor $\sim a^{4}$ for the processes with the neutrino participation and exponentially suppressed for reactions with antineutrino.

In the case of an ultrarelativistic electron-positron plasma, the neutrino- and antineutrinoproduction rates and the energy emitted in these processes depend not on the magnetic field strength itself but on dimensionless parameter $y_{b}=\sqrt{2 b} / t=\sqrt{2 e B} / T$ which contains the plasma temperature [12]:

$$
\begin{gathered}
\Gamma_{v}=\Gamma_{0} J_{2}\left(y_{b}, a\right) Y /(1+Y), \quad Q_{v}=Q_{0} J_{3}\left(y_{b}, a\right) Y /(1+Y), \\
\Gamma_{\bar{v}}=\Gamma_{0} J_{2}\left(y_{b},-a\right) /(1+Y), \quad Q_{\bar{v}}=Q_{0} J_{3}\left(y_{b},-a\right) /(1+Y), \\
\Gamma_{0}=\frac{\left(3 g_{a}^{2}+g_{v}^{2}\right) \cos ^{2} \theta_{c}}{8 \pi^{3}} G_{F}^{2} T^{5} \frac{\rho}{m_{N}}, \quad Q_{0}=T \Gamma_{0}, \\
J_{n}\left(y_{b}, a\right)=y_{b}^{2} \int_{0}^{y_{b}} \frac{y^{n} d y}{\exp (y-a)+1}+4 \int_{y_{b}}^{\infty} \frac{y^{n+2} d y}{\exp (y-a)+1} .
\end{gathered}
$$

Here, the values $\Gamma_{0} \approx 9.64 \times 10^{31} t^{5} \rho_{12} \mathrm{~cm}^{-3} \mathrm{~s}^{-1}$ and $Q_{0} \approx 7.88 \times 10^{25} t^{6} \rho_{12} \mathrm{erg} \mathrm{cm}^{-3} \mathrm{~s}^{-1}$ give the typical values for the neutrino- and antineutrino-production rates and the energy flux emitted 
in the beta-processes under conditions of the nondegenerate electron-positron plasma. Obviously, the function $J_{n}\left(y_{b}, a\right)$ introduced in (12) is quadratic in $y_{b}$ in the limit of the strong magnetic field $\left(y_{b} \gg a\right)$. So, $\Gamma_{v, \bar{v}}$ and $Q_{v, \bar{v}}$ must increase linearly with the growth of the magnetic field in this limit. However, in the case of a weak magnetic field, the rate of increase in the first term in $J_{n}\left(y_{b}, a\right)$ is lower than the rate of the decrease in the second term. Therefore, with the magnetic field increased, the beta-process rates and the energy emitted in these processes first decrease, come through their minimum values, and only then their linear increase begin as shown in Fig. 1 for the scaled reaction rates, $\Gamma_{v, \bar{v}}(b) / \Gamma_{v, \bar{v}}(0)$, and emitted energy, $Q_{v, \bar{v}}(b) / Q_{v, \bar{v}}(0)$. It can be seen that the minimum for the antineutrino-production rate
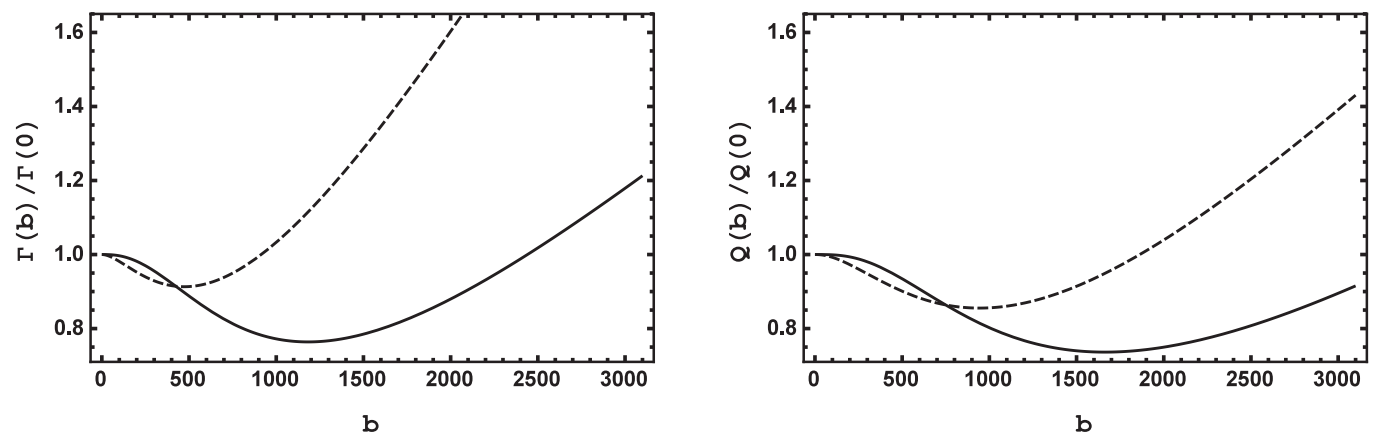

Figure 1. The beta-process rates (the left panel) and energy emitted through (anti)neutrino in these processes (the right panel) are plotted for the dimensionless temperature $t=8$ and electron degeneracy parameters $a=3$ as functions of the magnetic field $b=B / B_{0}$. The processes with the neutrino and antineutrino participation are shown by the solid and dashed curves, respectively.

and energy emitted in such reactions is not as deep as in the processes with neutrinos and is reached at lower values of the magnetic field strength.

Another important fact following from Fig. 1 should also be mentioned. A transition to an asymptotic linear increase for the beta-processes with antineutrinos occurs in lower magnetic fields in comparison to the reactions with neutrino. Nevertheless, the transition to asymptotic behavior even for these processes corresponds to the magnetic field with the strength $B \gtrsim 10^{16} \mathrm{G}$ which exceeds substantially the values typical for supernovae and accretion disks. Consequently, the increase in the magnetic field in these objects leads not to the intensification but conversely to the beta-process suppression which is manifested more strongly for the reactions with neutrinos. In addition, the suppression of the energy emitted is stronger than for the rates of the beta-processes.

Let us consider separately the effect of other parameters on the rates of the beta-processes and energy emitted in them. Numerical calculations show [12] that the dependence of these quantities on the temperature of the medium is similar for both reactions which is demonstrated in Fig. 2 for the relative energy $Q_{v, \bar{v}}(b) / Q_{v, \bar{v}}(0)$ emitted in neutrino and antineutrino. It can be seen that with the temperature increased, the position of the minimum of the relative energy emitted is shifted towards stronger magnetic fields. Its depth for each reaction separately remains unchanged but the depth of the minimum for the beta-processes with the neutrino participation is larger. It should be noted that the dependence of the reaction rates on the temperature of the medium is analogous to that shown in Fig. 2 but the depth of the relative minimum is slightly smaller for these quantities.

A different situation is observed in the dependence of the rates of the beta-processes and the energy emitted in them on the electron degeneracy parameter $a$. Numerical analysis has shown [12] that such 

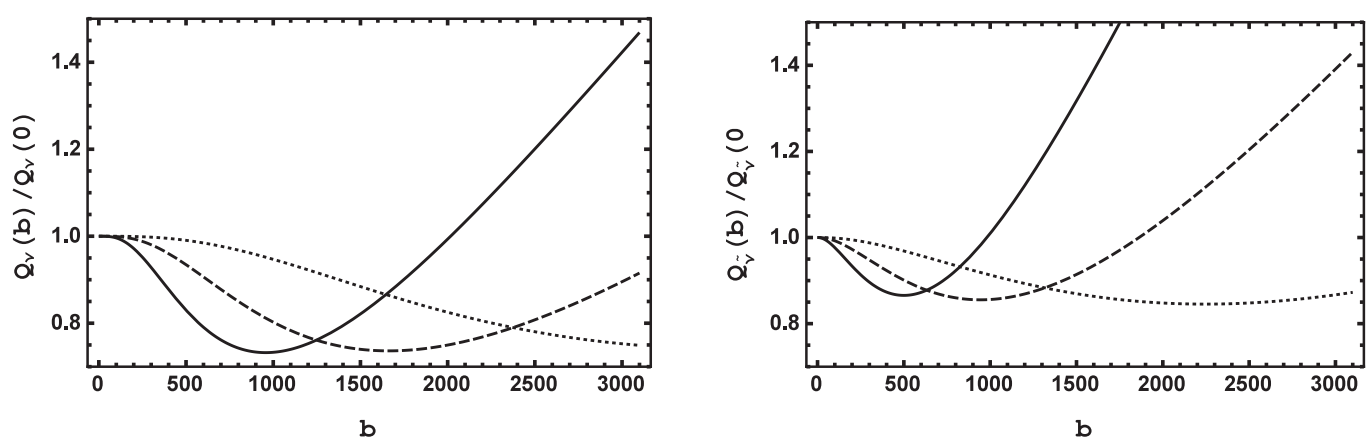

Figure 2. The relative energy emitted in neutrinos (the left panel) and antineutrinos (the right panel), corresponding to the electron degeneracy parameter $a=3$ and scaled temperatures $t=6$ (solid curves), $t=8$ (dashed curves), and $t=12$ (dotted curves) as functions of the magnetic field strength $b=B / B_{0}$.
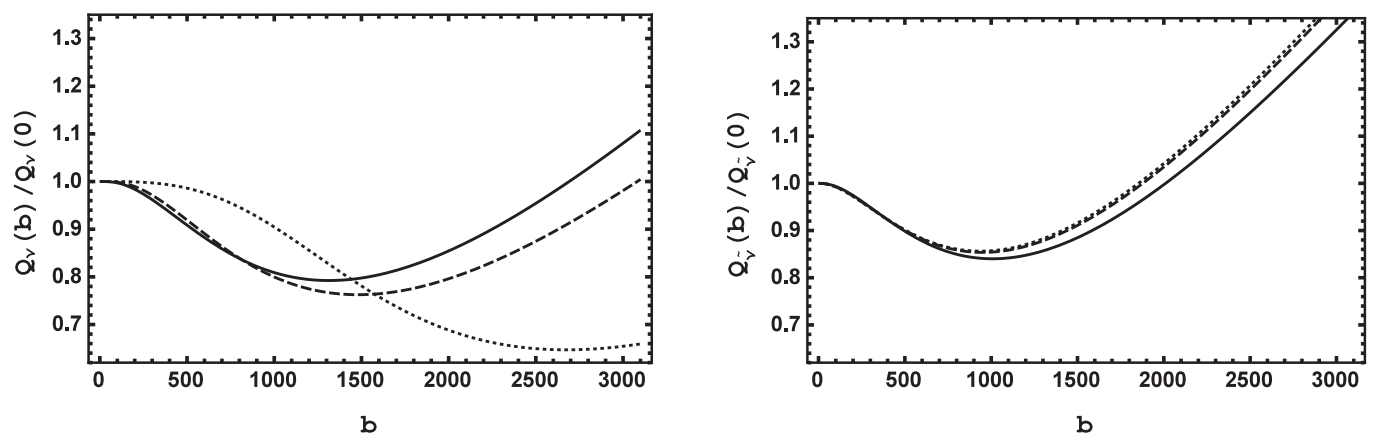

Figure 3. The relative energy emitted in neutrinos (the left panel) and antineutrinos (the right panel), corresponding to the scaled temperature $t=8$ and the electron degeneracy parameters $a=0$ (solid curves), $a=2$ (dashed curves), and $a=6$ (dotted curve) as functions of the magnetic field strength $b=B / B_{0}$.

dependences for the reactions with neutrino and antineutrino differ substantially as shown in Fig. 3 for the relative energy emitted, $Q_{v, \bar{v}}(b) / Q_{v, \bar{v}}(0)$. It can be seen from Fig. 3 that the maximum suppression of the reaction with the antineutrino participation is observed for the nondegenerate plasma $(a=0)$. However, the dependence of the relative energy emitted in these processes on the electron degeneracy parameter $a$ at a fixed temperature is very weak. The relative rate of the antineutrino generation demonstrates analogous behavior. It should be noted that this holds only for the relative quantities while the absolute values considerably depend on this parameter in terms of $\Gamma_{\bar{\nu}}(0)$ and $Q_{\bar{v}}(0)$. For the processes with neutrino, the situation is radically different because even relative values of the quantities strongly depend on the electron degeneracy parameter $a$. As seen in Fig. 3, the increase in this parameter leads to a displacement of the minima in the relative energy emitted in neutrino towards larger values of the field, and the minimum itself becomes deeper. The relative rate of the neutrino production behaves analogously but the depth of the minimum of this quantity is slightly smaller. Thus, the magnetic field suppresses the beta-processes with the neutrino participation the most strongly, and this suppression is the highest in a degenerate medium. 
In the case of the strong degeneracy of the electron-positron plasma, the following simple analytic expressions can be obtained for the integrals $J_{2}\left(y_{b}, a_{e}\right)$ and $J_{3}\left(y_{b}, a_{e}\right)$ [12]:

$$
\begin{aligned}
J_{2}\left(y_{b}, a_{e}\right) \approx 4 / 5 a_{e}^{5}-7 / 15 y_{b}^{5}, & J_{3}\left(y_{b}, a_{e}\right) \approx 2 / 3 a_{e}^{6}-5 / 12 y_{b}^{6}, \quad y_{b} \leqslant a_{e} \\
J_{2}\left(y_{b}, a_{e}\right) \approx 1 / 3 y_{b}^{2} a_{e}^{3}, & J_{3}\left(y_{b}, a_{e}\right) \approx 1 / 4 y_{b}^{2} a_{e}^{4}, \quad y_{b} \geqslant a_{e} .
\end{aligned}
$$

It can easily be seen that the relative neutrino production rate, $\Gamma_{v}\left(z_{b}\right) / \Gamma_{\nu}(0)$, and energy emitted, $Q_{v}\left(z_{b}\right) / Q_{v}(0)$, depend only on one variable $z_{b}=y_{b} / a_{e}=\sqrt{2 b} m_{e} / \mu_{e}$, and this dependence for the degenerate plasma is universal as regards $\mu_{e}$. The transition to such an asymptotic behavior is demonstrated in Fig. 4. The expressions obtained for $J_{n}\left(y_{b}, a_{e}\right)$ in the strongly degenerate plasma also show
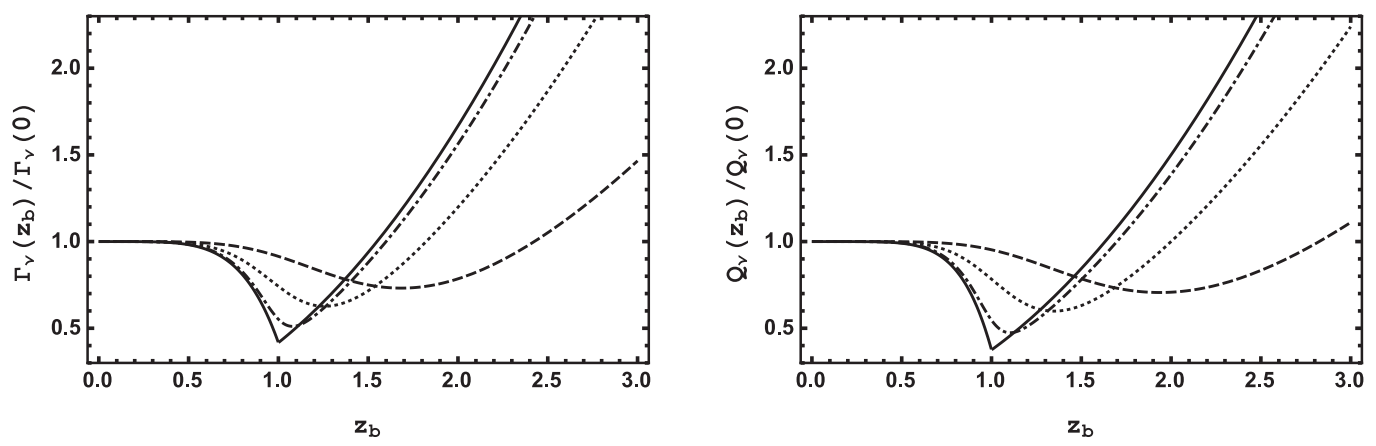

Figure 4. The relative rate of the neutrino production (the left panel) and energy emitted in this process (the right panel), corresponding to the scaled temperature $t=8$ and degeneracy parameters $a=4$ (dashed curves), $a=8$ (dotted curves), and $a=20$ (dash-dotted curves) as functions of the dimensionless parameter $z_{b}=\sqrt{2 e B} / \mu_{e}$. Solid curves correspond to the asymptotic form of the degenerate plasma.

that the largest suppression of the beta-processes with neutrino by the magnetic field occurs for the strength $B_{v} \approx \mu_{e}^{2} /\left(2 m_{e}^{2}\right) B_{0}$, and the magnitude of the suppression relative to the field-free value is $\Gamma_{v}\left(B_{v}\right) / \Gamma_{v}(0)=5 / 12 \approx 0.42$ for the neutrino-production rate and $Q_{v}\left(B_{v}\right) / Q_{v}(0)=3 / 8 \approx 0.38$ for the energy emitted.

For the beta-processes with the antineutrino participation, the convenient variable is the dimensionless parameter $y_{b}=\sqrt{2 b} / t$. Numerical calculations show [12] that the relative rate of the antineutrino production, $\Gamma_{\bar{v}}\left(y_{b}\right) / \Gamma_{\bar{v}}(0)$, and energy, $Q_{\bar{v}}\left(y_{b}\right) / Q_{\bar{v}}(0)$, emitted in such processes as functions of this variable weakly depend on the temperature of the medium and the plasma degeneracy parameter as shown in Fig. (5). The same figure depicts the asymptotic form of the nondegenerate $\left(a_{e}=0\right)$ ultrarelativistic plasma in which, as noted above, the suppression this process by the magnetic field is the strongest. It follows from the results of numerical calculations [12] that the maximum suppression of the rate of the antineutrino production occurs in the magnetic field with the strength $B_{\Gamma_{\bar{v}}} \approx 10 t^{2} B_{0}$, and the magnitude of the suppression in this case is $\Gamma_{\bar{v}}\left(B_{\Gamma_{\bar{\nu}}}\right) / \Gamma_{\bar{v}}(0) \simeq 0.86$. The maximum suppression of the energy emitted in antineutrinos is shifted towards stronger fields, $B_{Q_{\bar{v}}} \approx 18 t^{2} B_{0}$, and the suppression itself is slightly stronger, $Q_{\bar{v}}\left(B_{Q_{\bar{v}}}\right) / Q_{\bar{v}}(0) \simeq 0.82$. Comparison with analogous quantities obtained for processes with the neutrino participation shows that the maximum suppression of these reactions with the neutrino participation by the magnetic field occurs in the nondegenerate medium, and the suppression itself is approximately half as strong. 

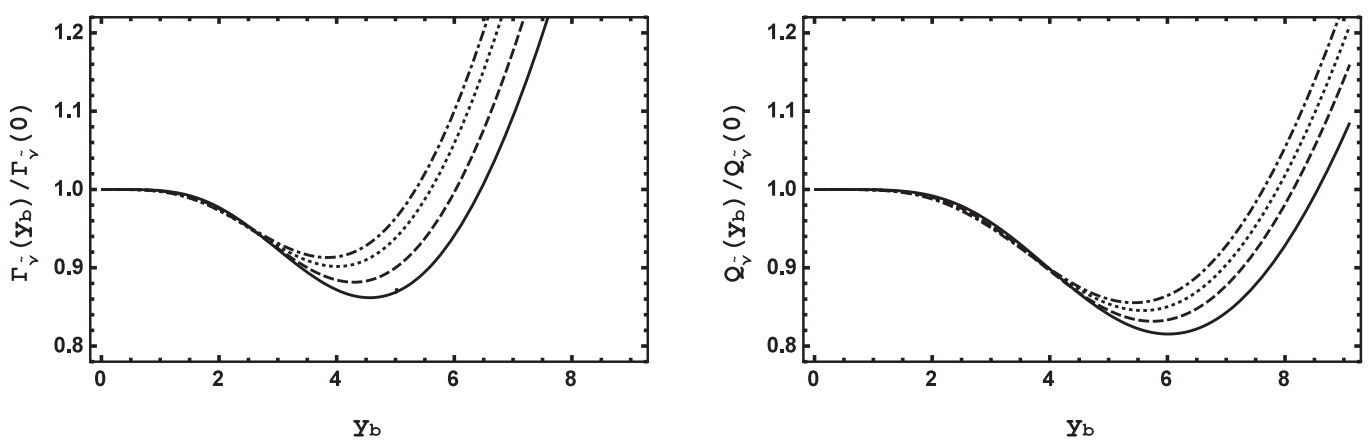

Figure 5. The relative rate of the antineutrino production (the left panel) and energy emitted in this process (the right panel) as functions of the dimensionless parameter $y_{b}=\sqrt{2 b} / t$. Solid curves correspond to the asymptotic form of an ultrarelativistic nondegenerate plasma $(a=0)$ in which process $(2)$ has the most strong suppression by the magnetic field. The remaining curves correspond to the following dimensionless parameters: $t=12$ and $a=0$ (dashed curves), $t=12$ and $a=3$ (dotted curves), and $t=8$ and $a=3$ (dash-dotted curves).

\section{Conclusions}

In this paper, we have analyzed the beta-processes (1)-(3) in the medium of supernovae and accretion disks with an arbitrary magnitude of the magnetic field. The results obtained for the transparent medium were used to calculate the neutrino and antineutrino production rates, the energy emitted in these processes, and the macroscopic momentum transferred to the medium.

The simplest dependence on the magnetic field was obtained for the macroscopic momentum transferred to the matter. It was shown that in the case of an transparent medium, it increases linearly with the magnetic field and is directed along the field-strength lines. It was demonstrated that this effect is most significant in the degenerate electron-positron plasma. Unlike the momentum transferred, the rates of the beta-processes and energy emitted in them exhibit a more complex dependence on the magnetic field. In the limit of the strong magnetic field, these quantities, as well as the momentum transferred, increase linearly with the growth of the magnetic field. It was shown, however, that for the values $B \lesssim 10^{15} \mathrm{G}$ which are typical for supernovae and accretion disks, the values of these quantities are suppressed as compared to the field-free case. The maximum suppression of the reactions with the neutrino participation occurs in the degenerate medium in the field with the strength $B_{v} \sim \mu_{e}^{2} /\left(2 m_{e}^{2}\right) B_{0}$ which falls into the range of values typical of the objects studied. The suppression can reach 2.5 times as compared to the field-free case. The effect of the magnetic field on the antineutrino processes is weaker and its maximum suppression is 1.2 times as compared to the field-free case. However, such a suppression occurs in the nondegenerate plasma (i. e., in regions of supernovae and accretion disks with a moderate density). The magnetic field corresponding to the maximum suppression of these processes is $B_{\bar{v}} \sim 10 t^{2} B_{0}$ which also falls in the range of values typical of the objects considered at moderate temperatures. Thus, the suppression of the processes with the neutrino and antineutrino participation, by the magnetic field occurs in different parts of supernovae and accretion disks, and the effect itself can be significant.

It should be noted that the form of the dependence of the rates of the beta-processes and the energy-momentum transferred in these processes on the magnetic field and parameters of the matter presented here does not change significantly in the more general case of a medium partly transparent to neutrinos and antineutrinos. 
I'd like thank to Thomas Janka for numerous discussions on this topic and possible extensions of this research. It is a pleasure to thank G. S. Bisnovatyi-Kogan, Alexander Parkhomenko and Alexandra Dobrynina for stimulating discussions and critical comments. This study was partially supported by the Russian Foundation for Basic Research (project no. 15-02-06033-a) and by the Michael Lomonosov Program (project no. 1.721.2016/2.2).

\section{References}

[1] P. Kumar, B. Zhang, Phys. Rept. 561, 1 (2014), 1410.0679

[2] A.I. MacFadyen, S.E. Woosley, Astrophys. J. 524, 262 (1999), astro-ph/9810274

[3] S. Rosswog, Int. J. Mod. Phys. D24, 1530012 (2015), 1501.02081

[4] M.V. Barkov, S.S. Komissarov, Mon. Not. Roy. Astron. Soc. 401, 1644 (2010), 0908.0695

[5] S.W. Bruenn, Astrophys. J. Suppl. 58, 771 (1985)

[6] S.G. Moiseenko, G.S. Bisnovatyi-Kogan, Astron. Rep. 59, 573 (2015)

[7] H. Sawai, S. Yamada, Astrophys. J. 817, 153 (2016), 1504.03035

[8] I. Zalamea, A.M. Beloborodov, Mon. Not. Roy. Astron. Soc. 410, 2302 (2011), 1003.0710

[9] A.A. Gvozdev, I.S. Ognev, JETP Lett. 74, 298 (2001), [Pisma Zh. Eksp. Teor. Fiz. 74, 330 (2001)], astro-ph/0201346

[10] A.A. Gvozdev, I.S. Ognev, J. Exp. Theor. Phys. 94, 1043 (2002), [Zh. Eksp. Teor. Fiz. 121, 1219 (2002)], astro-ph/0403011

[11] A.A. Gvozdev, I.S. Ognev, Astron. Lett. 31, 442 (2005)

[12] I.S. Ognev, J. Exp. Theor. Phys. 123, 643 (2016), [Zh. Eksp. Teor. Fiz. 150, 744 (2016)]

[13] C. Patrignani et al. (Particle Data Group), Chin. Phys. C40, 100001 (2016) 